\title{
A Systematic Review of the Efficacy of Repetitive Transcranial Magnetic Stimulation Treatment for Women with Postpartum Depression
}

\author{
Maddison M. Huddle 1,2,*, Shane C. Costello ${ }^{1,2}$ and David A. Barton $2,3,4$ \\ 1 Faculty of Education, Monash University, Clayton, VIC 3800, Australia; shane.costello@monash.edu \\ 2 NeuroCentrix Research Institute, Noble Park, VIC 3174, Australia; david@neurocentrix.com.au \\ 3 Australian Centre for Heart Health, North Melbourne, VIC 3051, Australia \\ 4 South-Eastern Private Hospital, Noble Park, VIC 3174, Australia \\ * Correspondence: maddison.huddle@monash.edu
}

Citation: Huddle, M.M.; Costello, S.C.; Barton, D.A. A Systematic Review of the Efficacy of Repetitive Transcranial Magnetic Stimulation Treatment for Women with Postpartum Depression. Psychiatry Int. 2021, 2, 265-276. https://

doi.org/10.3390/psychiatryint2030020

Academic Editor: Takahiro A. Kato

Received: 7 May 2021

Accepted: 16 June 2021

Published: 1 July 2021

Publisher's Note: MDPI stays neutral with regard to jurisdictional claims in published maps and institutional affiliations.

Copyright: (C) 2021 by the authors. Licensee MDPI, Basel, Switzerland. This article is an open access article distributed under the terms and conditions of the Creative Commons Attribution (CC BY) license (https:// creativecommons.org/licenses/by/ $4.0 /)$.

\begin{abstract}
Transcranial Magnetic Stimulation (TMS) has a strong evidence base for the treatment of major depressive disorder (MDD), however, there is minimal research investigating the treatment of depression within the postpartum period. This systematic review aims to systematically examine the efficacy and safety of TMS when treating postpartum depression (PPD). Databases Ovid Medline, PsycINFO, EMBASE and PubMed were searched from inception to May 2021, to identify peer-reviewed papers assessing the administration of TMS for PPD treatment. Data were systematically extracted and evaluated regarding clinical psychiatric outcomes, social-relational outcomes, neuropsychological testing, and side effects. This systematic review included one randomised controlled trial, two open-label studies, two conference papers, and two case studies, providing data on 60 participants. TMS appears well tolerated, with no reported major adverse side effects. While the studies reported a general reduction in PPD symptoms, the poor quality of the evidence available indicates that TMS for PPD is unable to be currently recommended. However, the substantial evidence for TMS in the treatment of MDD and the differential response to antidepressant medication in PPD indicates that further research into TMS for PPD is warranted.
\end{abstract}

Keywords: postpartum depression; major depression; systematic review; women mental health; DLPFC

\section{Introduction}

Women experiencing significant postpartum depression (PPD) require safe and effective treatment for depressive symptoms. Research has estimated 11-20\% of new mothers experience minor and 7-14\% major depression after the delivery of their baby [1-3]. PPD not only affects the mother but can also have significant deleterious impacts on the child, with effects ranging from adverse maternal-infant bonding to infanticide and suicide [4]. Currently, non-pharmacological and non-invasive brain stimulation (NIBS) techniques, such as Transcranial Magnetic Stimulation (TMS) have been recognised as an option for PPD treatment, as it can manage certain limitations of more traditional methods [5].

PPD is defined by the Diagnostic and Statistical Manual of Mental Disorders-5 [6] as a Major Depressive Disorder (MDD), with the onset of the most recent episode occurring during pregnancy (prenatal) or within the four weeks after childbirth (postnatal). However, existing research, clinical practice and epidemiological studies report the postpartum period involves 12 months after childbirth [7,8].

Electroconvulsive therapy (ECT), psychotherapy, pharmacotherapy, or a combination of these evidence-based treatments are used for moderate-severe PPD. ECT is reported to be clinically effective, however, it requires anaesthesia and causes muscle pain, and temporary disorientation and memory impairment $[9,10]$. Psychotherapy presents comparative efficacy [11,12] with medium to large effect sizes [13]; however, is not effective for all 
women with PPD [14], and is typically not adequate for severe depression [15]. Moreover, mothers can be reluctant to engage due to fears of being seen as inadequate parent [11].

Meta-analyses on pharmacotherapy for PPD treatment are inconclusive [16,17], however clinical consensus indicates Selective Serotonin Reuptake Inhibitors (SSRIs) are used as the first line of treatment. Some research proposes that SSRIs demonstrate reasonable efficacy rates (50-67\%) and treatment response is between the 6th and 8th week [18]. Moreover, the latest expert opinions report a relative low risk of using Sertraline whilst breastfeeding [19]. Despite the apparent efficacy of SSRIs, studies show that physicians resist prescribing them to breastfeeding women, or prescribe doses below effective levels [18].

In addition to practitioner's hesitancy to prescribe SSRIs, breastfeeding women with depression are less likely to take medication $[20,21]$. Women report concerns about medication side effects, particularly weight gain, decreased libido, and cognitive clouding with antidepressants $[22,23]$. They also express significant concern regarding the safety of a breastfed infant on the rapidly developing brain [24]. This is problematic as breastfeeding is a vital component of early parenting and a positive relationship with the infant is essential to recovery from PPD.

The treatment of PPD is laden with numerous complexities. Women typically have more severe symptoms of depression and tend to present with co-morbid anxiety, as opposed to men $[25,26]$. The sex difference in the incidence of depression is greatest during the reproductive years, indicating that sex hormones and reproductive events play some role in the aetiology of depression [26-29]. This suggests depression within the postpartum period differentiates from depression outside the postpartum period. Therefore, different treatment options need to be considered for this population.

Research on non-invasive brain stimulation (NIBS) techniques have significantly developed practitioner's understanding of brain-behaviour relationships over the past three decades. NIBS techniques enable safe modulation of neural processes in the brain, to allow researchers to directly study how alternated neural activity affects behaviour [30]. NIBS methods are broadly used in the research and treatment of different psychiatric disorders, such as post-traumatic stress disorder (PTSD) [31], anxiety and phobia [32-34] and obsessive-compulsive disorder (OCD) $[35,36]$. TMS has emerged as one of the key mainstays of NIBS and increased in popularity over the past 20 years [37], particularly involving the treatment of MDD.

TMS is a non-invasive, non-systemic device that administers pulsed, magnetic fields to provoke an electric current in the cerebral cortex [38]. When pulses of TMS are delivered repetitively, this is called repetitive TMS, or rTMS. The proposed aim of rTMS is to either stimulate or inhibit regions of the neural cortex [39]. Studies have found lower magnetic frequencies $(\leq 1 \mathrm{~Hz})$ create an inhibitory effect, and higher frequencies $(>5 \mathrm{~Hz})$ create an enhancing effect [39-43]. The meta-analysis of imaging studies and rTMS by Taib et al. [39] reports there are multiple brain structures involved in the pathophysiology of MDD including; amygdala; dorsolateral prefrontal cortex (DLPFC); ventrolateral and ventromedial PFC; hippocampus; the anterior cingulate cortex (ACC); inferior frontal cortex; and basal ganglia. Taib et al. [39] explain the structures involved in rTMS treatment correspond to a number of those implicated in depression.

Research has shown rTMS to modify areas of the brain, however, the efficacy of rTMS appears related to the brain stimulate site; the primary mechanisms from the stimulation, instigating these brain modifications, remains unconfirmed [39]. Despite the limited understanding of the exact mechanisms involved, multiple neuroimaging studies on MDD present hypoactivity of the left DLPFC and hyperactivity of the right DLPFC [39]. Hence, practitioners are applying the inhibiting or enhancing properties of rTMS to module dysfunction in these brain structures.

Current research has demonstrated that two different protocols are effective in the reduction or remission of depressive symptoms: low-frequency right DLPFC rTMS, or highfrequency left DLPFC rTMS $[39,44]$. Bi-lateral rTMS is an acceptable alternative treatment if previous protocols do not produce the preferred outcomes for patients [44,45]. Bi- 
lateral rTMS involves both the left and right DLPFC to be stimulated either sequentially or simultaneously [45]. Prefrontal TMS therapy repeated daily over 4-6 weeks (20-30 sessions) is approved by the US Food and Drug Administration (FDA) for treating MDD in adults who have not responded to prior antidepressant medications [46].

More than 30 randomized controlled trials, including over 2000 participants have been produced to investigate the efficacy of TMS in treating MDD [38]. The data from these studies have been examined and reviewed in over 10 meta-analyses and two qualitative reviews $[47,48]$. Notwithstanding the increasing amount of research on TMS treatment for depressive symptoms, there is limited data on the efficacy, safety and acceptability of this treatment for women with PPD [49]. Studies have found that PPD differentiates from MDD outside the postpartum period, hence additional research is required for PPD treatment with TMS. Current biological treatments are limited due to concerns over efficacy, long term effects on the breastfeeding infant and side effects which impact upon compliance [50]. The objective of the current systematic review is to assess the efficacy of TMS for women with PPD.

\section{Materials and Methods}

This systematic review employed the 'Preferred Reporting Items for Systematic Reviews and Meta-Analyses' (PRISMA) model [51]. This paper aimed to identify all published reports of data-based empirical studies, to explore the efficacy of TMS for depression treatment in patients with PPD. To obtain the maximum number of studies for inclusion, no restrictions were applied to the study sample size, year of study or study quality.

\subsection{Search Strategy}

An initial search was conducted to identify the quantity of existing primary research and determine what search terms would be effective in finding appropriate articles. Searches were conducted across four databases (Ovid Medline, PsycINFO, EMBASE and PubMed). The following search terms were applied to find relevant studies; "depress"”, "*partum", "*natal", "transcranial magnetic stimulation", "repetitive transcranial stimulation", "TMS", "rTMS". Searches within databases were restricted to "full-text," "peer-reviewed" and "English". The search was completed on 6 May 2021.

\subsection{Study Selection}

Fifty papers were identified in the primary searches and entered into EndNote, which assisted in the screening of papers by title and abstract. Duplicate entries were removed, resulting in 22 records for screening in accordance with the inclusion/exclusion criteria.

The primary and secondary authors independently screened all 21 records by title and abstract. Papers were excluded if studies focused on depression outside of the postpartum period; articles published in a language other than English, or details of the research were incomplete or insufficient. Articles without empirical data (such as opinion pieces, viewpoints or editorials) were also removed, leaving a total of six articles to be read in full. The reference lists of all identified papers, as well as relevant reviews, were hand searched, resulting in the inclusion of one additional paper. Full-text screening was conducted on the seven identified papers by the primary author. Each paper met the inclusion criterion for the systematic review. A diagram of the full search and screening processes are shown in Figure 1. 


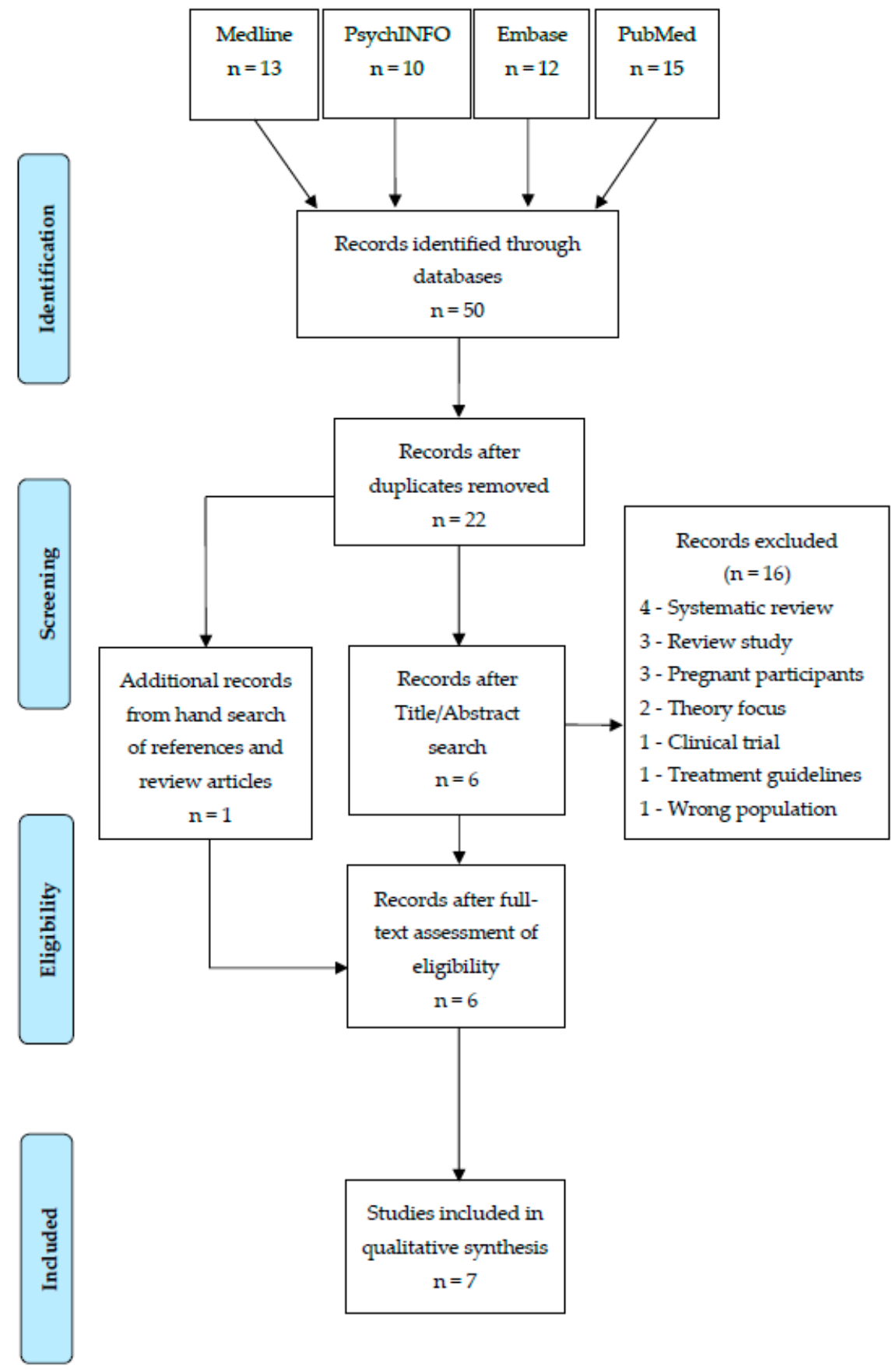

Figure 1. Study Selection PRISMA flow diagram.

\subsection{Quality Assessment}

The Crowe Critical Appraisal Tool (CCAT) [52] and the accompanying guide for evaluation of studies in a systematic review [53], were employed to assess the quality of the included papers. CCAT is designed for health research and can be utilised for multiple study designs $[54,55]$. The research design, variables and analysis, sampling, and data collection were outlined using the CCAT. Scores were determined for each category item, including; preliminaries, introduction, design, sampling, data collection, ethical matters, results, and discussion. This tool also assessed the risk of bias for included studies. Total scores were rated out of 40 , which were converted into a percentage. Table 1 provides an overview of the seven studies with their respective quality rating. CCAT forms for each included study can be found in the supplementary materials. 
Table 1. Overview of findings from 7 papers including participants and study methodologies.

\begin{tabular}{|c|c|c|c|c|c|c|c|c|c|c|c|c|c|}
\hline Study & $\begin{array}{l}\text { Quality } \\
\text { Rating }\end{array}$ & $\mathrm{N}$ & $\begin{array}{r}\text { Participants } \\
\text { Age * }\end{array}$ & $\begin{array}{c}\text { Study } \\
\text { Design }\end{array}$ & $\begin{array}{c}\text { Stimulation } \\
\text { Site }\end{array}$ & $\begin{array}{c}\text { Frequency } \\
(\mathrm{Hz})\end{array}$ & $\begin{array}{c}\text { No. of } \\
\text { Pulses }\end{array}$ & $\begin{array}{c}\text { No. of } \\
\text { Trains ITI }\end{array}$ & $\begin{array}{l}\text { Intensity } \\
(\% \mathrm{RMT})\end{array}$ & $\begin{array}{c}\text { No. of } \\
\text { Sessions }\end{array}$ & $\begin{array}{l}\text { Outcome } \\
\text { Measures }\end{array}$ & Time-Points & Outcomes \\
\hline $\begin{array}{c}\text { Brock et al. } \\
(2016)\end{array}$ & $20 \%$ & 19 & & Conference paper & Left DLPFC & - & & - & - & & EPDS & Baseline, week 8 & $\begin{array}{l}\text { Reduction in depressive symptoms. Follow up scores remained below } \\
\text { baseline scores. } 73.7 \% \text { of patients achieved remission of their depressive } \\
\text { symptoms with acute treatment. }\end{array}$ \\
\hline $\begin{array}{l}\text { Cox et al. } \\
(2020)\end{array}$ & $67.5 \%$ & 6 & 33.5 (30-41 years) & Open-label & Left DLPFC & 10 & 3000 & $\begin{array}{l}75 \\
26 s\end{array}$ & 120 & 20 & $\begin{aligned} \text { BDI } \\
\text { EPDS } \\
\text { STAI }\end{aligned}$ & $\begin{array}{l}\text { Baseline, weekly, and } 3 \\
\text { and } 6 \text { months follow up }\end{array}$ & $\begin{array}{l}\text { A general reduction in symptoms however variable across outcome } \\
\text { measures. Follow up scores remained below baseline scores. }\end{array}$ \\
\hline \multirow{2}{*}{$\begin{array}{l}\text { Cohen et al. } \\
(2008) \text {. }\end{array}$} & \multirow{2}{*}{$50 \%$} & \multirow{2}{*}{1} & \multirow{2}{*}{36 years } & \multirow{2}{*}{ Case study } & Right DLPFC & 20 & 1600 & - & 100 & 1 & \multirow{2}{*}{$\begin{array}{l}\text { HDRS } \\
\text { YMRS }\end{array}$} & \multirow{2}{*}{$\begin{array}{l}\text { Baseline, weekly, and } 1 \\
\text { month follow up }\end{array}$} & \multirow{2}{*}{$\begin{array}{l}\text { Reduction of mania symptoms after right DLPFC Tx; however, returned } \\
\text { symptoms of depression: sadness, depressed mood, feelings of guilt, and } \\
\text { difficulty concentrating with no psychotic symptoms. Post bilateral Ix, } \\
\text { reduction of both depression and manic symptoms. }\end{array}$} \\
\hline & & & & & Bilateral DLPFC & 20 & $\begin{array}{l}800 \text { right, } \\
800 \text { left }\end{array}$ & - & 100 & 9 & & & \\
\hline $\begin{array}{c}\text { Garcia et al. } \\
(2010) .\end{array}$ & $65 \%$ & 9 & $34.11 \pm 6.05$ & Open-label & Left DLPFC & 10 & 3000 & $\begin{array}{l}75 \\
26 \mathrm{~s}\end{array}$ & 120 & 20 & $\begin{array}{l}\text { HRDS-24 } \\
\text { EPDS } \\
\text { IDS-SR } \\
\text { CGI-S } \\
\end{array}$ & $\begin{array}{l}\text { Baseline, end of week 2, } \\
\text { end of week } 4, \text { and } \\
\text { 180-day follow-up }\end{array}$ & $\begin{array}{l}\text { General reduction in depressive symptoms. } 88.9 \% \text { of participants achieved } \\
\text { remission of symptoms. Results also ondicacted a significant improvement } \\
\text { in bonding. }\end{array}$ \\
\hline $\begin{array}{l}\text { Myczkowski } \\
\text { et al. (2012). }\end{array}$ & $72.5 \%$ & 14 & 28.5 (18-36 years) & RCT & $\begin{array}{l}\text { Left DLPFC } \\
\text { Sham }\end{array}$ & 5 & 1250 & $\begin{array}{l}25 \\
20 \text { s }\end{array}$ & 120 & 20 & $\begin{array}{c}\text { HDRS-17 } \\
\text { EPDS } \\
\text { HARS-14 } \\
\text { CGI-1,-2,-3 } \\
\text { GA } \\
\text { SF-36 } \\
\text { SAS } \\
\text { NAB } \\
\end{array}$ & $\begin{array}{l}\text { Baseline, } 4 \text { weeks of } \\
\text { treatment, week } 6 \text { follow } \\
\text { up }\end{array}$ & $\begin{array}{l}\text { General reduction in symptoms however variable across outcome } \\
\text { measures. Follow up scores remained below baseline scores. }\end{array}$ \\
\hline $\begin{array}{c}\text { Ogden et al. } \\
\text { (1999). }\end{array}$ & $40 \%$ & 1 & 40 years & Case study & Left DLPFC & 20 & 1200 & $\begin{array}{l}30 \\
28 \mathrm{~s}\end{array}$ & 100 & 13 & $\begin{array}{c}\text { HDRS } \\
\text { BDI } \\
\text { VAS }\end{array}$ & Baseline, end of treatment & $\begin{array}{l}\text { General reduction in symptoms however Pt complained of low energy and } \\
\text { motivation, lack of enjoyment and poor sleep a week after discharge. } \\
\text { Citatopram dose was increased to } 40 \mathrm{mg} \text { g daily. Follow up revealed no } \\
\text { symptoms of depression and improved family relationships. }\end{array}$ \\
\hline $\begin{array}{c}\text { Ozmutet al. } \\
(2015)\end{array}$ & $17.5 \%$ & 15 & - & Conference paper & - & 20 & 1000 & $50 \mathrm{~s}$ & 80 & 15 & $\begin{array}{l}\text { HDRS-17 HARS-14 } \\
\text { EDS-10 }\end{array}$ & Sessions $1,7,15$ & $\begin{array}{l}\text { Less than half of participants achieved a general reduction in symptoms, } \\
\text { however variable across outcome measures. }\end{array}$ \\
\hline
\end{tabular}




\subsection{Data Analysis}

Key information was extracted from each of the studies including authors, year of publication, number and age of participants, study design, outcome measures and timepoints, and key findings (in relation to the research questions of this review). Details of TMS protocols within each paper were also included where possible (e.g., stimulation site, frequency, number of pulses and trains, inter-train interval, intensity and number of sessions).

\section{Results}

\subsection{Description of Studies}

The seven papers within this systematic review included one randomised controlled trial [50], two open-label studies [4,56], two conference papers [57,58], and two case studies $[59,60]$, providing data on 60 participants, whereby 54 were treated with active-TMS and 6 were treated with sham-TMS (an inactive form of stimulation used to control for the placebo effect) [61]. Characteristics from each of the selected papers are displayed in Table 1.

Six of the seven of the studies focused on treatment for patients with PPD $[4,50,56-58,60]$. One of those studies involved a patient diagnosed with PPD with psychotic features [60]. The remaining study involved a patient diagnosed with bipolar disorder with depressive episodes in the postpartum period [59].

Most TMS protocols involved stimulation over the left dorsolateral prefrontal cortex (DLPFC) $[4,50,56,57,60]$. Of these studies, one paper administered TMS using a frequency of $20 \mathrm{~Hz}$ [60]; two papers used a frequency of $10 \mathrm{~Hz}$ [4,56], and one paper used a frequency of $5 \mathrm{~Hz}$ [50]. This paper also included a control group of sham TMS. The paper by Brock et al. [57] did not specify the frequency used within their TMS protocol. One study involved a single session of right DLPFC TMS at a frequency of $20 \mathrm{~Hz}$ [59]. This paper also involved nine sessions of bilaterally stimulating the left and right side of the DLPFC at a frequency of $20 \mathrm{~Hz}$. The remaining study by Ozmut et al. [58] did not specify which area of the brain was stimulated during their TMS protocol; however, investigators did state they used a frequency of $20 \mathrm{~Hz}$. Three studies administered a stimulus intensity of $120 \%$ of the resting motor threshold (RMT) $[4,50,56]$. Two studies administered $100 \%$ of the RMT [59,60], one study administered $80 \%$ of the RMT [58], and one study did not specify the stimulus intensity of the RMT [57]. Across the seven papers included, there was variation in the number of treatments given to patients. Two studies involved 20 sessions of treatment [4,56], one study involved 15 sessions [58], one study involved 13 sessions of treatment [60], one study involved 10 sessions of treatment (one session of right-sided treatment, nine sessions of bilateral treatment) [59], and one study did not report the number of TMS treatments given [57].

\subsection{Clinical Psychiatric Outcomes}

The majority of papers found a general reduction in PPD symptoms; however, scores varied across different outcome measures $[4,50,56,57,60]$. Follow up scores of these studies consistently remained below baseline scores. Cox et al. [56] found Beck Depression Inventory (BDI) [62], Edinburgh Postnatal Depression Scale (EPDS) [63], and State-Trait Anxiety Inventory (STAI) [64] scores declined over the 4-week duration of TMS treatment. Of the six patients, four achieved remission as assessed by EPDS and one achieved remission and two responded as assessed by BDI. Mean BDI and EPDS scores at 3- and 6-months follow-up remained below levels at study entry. Brock et al. [57] reported the EPDS mean baseline score was $20.6(\mathrm{SD}=4.15)$ compared with the mean end of acute treatment score of $8.2(\mathrm{SD}=6.50)$. Fourteen out of nineteen participants achieved remission of their depressive symptoms (EPDS < 10). Similarly, Garcia et al. [4] reported significant improvement in depression symptomatology at each timepoint. After the acute course of 20 TMS sessions, eight out of nine participants achieved remission of symptoms, defined as a Hamilton Rating Scale for Depression (HRSD-24) [65] <10 and a Clinical Global Impressions (CGI) [66] 
'Severity' subscale $=1$. At 6-month follow up, of the eight that remitted, seven remained in remission without further psychiatric intervention.

Myczkowski et al. [50] found the active TMS group presented statistically significant differences in scores at base rate versus week 6 for the Hamilton Depression Rating Scale (HDRS-17) [67], Global Assessment Scale (GAS) [68], and CGI for 'Severity' outcome measures. The active and sham groups both indicated a reduction in PPD symptoms, however, reduction percentages were notably larger for the active group when comparing baseline to week 6 of treatment; with respect to EPDS (39.4\% versus 6.2\%), Hamilton Anxiety Rating Scale (HARS-14; [69] 8.1\% versus 6.6\%), CGI 'Quality of Life' subscale (CGI3; $44.2 \%$ versus $26.3 \%$ ), Short-Form Health Survey (SF-36) [70] for vitality $(80.0 \%$ versus $17.9 \%$ ) and mental health (102.3\% versus $8.6 \%)$. Nonetheless, results were not deemed statistically significant when comparing active versus sham TMS. Odgen et al. [60] also found improvements in outcome measures at baseline versus completion of treatment; HDRS score reduced from 29 to 3, the BDI from 48 to 13 and the Visual Analogue Scale (VAS) [71] from 8.2 to 3.4. The VAS is used to measure the intensity and/or frequency of depressive symptoms [60].

Cohen et al. [59] initially administered right DLPFC TMS due to their patient's presentation of mania. After a single session, researchers found a reduction in mania symptoms; however, three days later the patient returned with symptoms of depression (Young Mania Rating Scale; YMRS [72] = 10, HDRS = 22). As a result, bilateral high-frequency TMS was administered for nine sessions to manage the patient's rapid cycling episode. Outcome measures indicated excellent improvement of both depression and manic symptoms (YMRS, HDRS $<5$ ) at the end of treatment.

The paper by Ozmut et al. [58] was the only study where the majority of participants had a less promising response to treatment. Out of a total of fifteen participants, four reported a 50\% decrease in HDRS-17 scores, six reported a 50\% decrease in HARS-14 scores, and eight reported Edinburgh Depression Scale (EDS-10) [63] scores of less than 13 points following treatment. This paper also included a combination of participants with major depression during pregnancy and postpartum and did not specify in the results how each group responded and instead provided a general statement about all participants.

\subsection{Social-Relational Outcomes}

Two studies reported on participants' ability to bond with their baby pre and post TMS treatment $[4,60]$. The study by Garcia et al. [4] found there was a statistically significant improvement in Postpartum Bonding Questionnaire (PBQ) [73,74] scores from baseline to post-treatment assessment. Ogden et al. [60] also presented a case study that identified improvement in the mother-baby relationship. Researchers reported the patient had attempted infanticide pre-treatment. The patient experienced depressive cognitions about herself and the child, suicidal ideation, and rejected contact with the child. After one week of treatment, the patient no longer rejected the idea of visiting her child. Two months follow up revealed the mother and her child were enjoying a 'close relationship' (p. 3).

One study investigated the participants' social performance at baseline and post TMS treatment [50]. This paper found the active TMS group had improved scores on the Social Adjustment Scale Self-Report (SAS-SR) [75] by $25.5 \%$ from baseline to week 6, whereas the sham group had only improved by $3.8 \%$. However, these results were not statistically significant. Nonetheless, a statistically significant improvement was observed both at baseline to week 4 (end of treatment) and baseline to week 6 ( 2 weeks after treatment) for the SAS-SR 'Work at Home' subscale.

\subsection{Neuropsychological Testing}

Studies by Cox et al. [56] and Myczkowski et al. [50] assessed participants neurocognitive performance at baseline and post TMS treatment. Cox et al. [56] found no changes across the Mini-Mental State Examination (MMSE) [76], Trail Making Test-B (TMT-B) [77] or Word List Generation (WLG) [78] cognitive assessments. Myczkowski et al. [50] also 
found their scores were not statistically significant when comparing active versus sham TMS. However, reduction percentages were larger for the active group when comparing baseline to post treatment; with respect to TMT-B (31.4\% versus $12.9 \%)$ and Victoria Stroop Test-Interference [79] (31.7\% versus 10.0\%).

\subsection{Side Effects}

Three studies reported no adverse side effects from TMS treatment $[57,58,60]$. Three studies mentioned minor side effects such as scalp discomfort and/or headache $[4,50,56]$. The paper by Garcia et al. [4] also reported, out of nine participants, eight preferred TMS to medication; however, only six believed it was convenient. The remaining study did not describe any side effects from TMS treatment [59].

\section{Discussions}

This systematic review gathered evidence for the efficacy of TMS in treating PPD. The treatment of PPD is a high public health priority, with approximately $7-14 \%$ of new mothers experiencing major depression after giving birth [1-3]. Traditional methods of treatment for PPD present their own disadvantages and inadequately account for the complexity of PPD. Although effective, ECT presents with a number of adverse side effects $[9,10]$. Psychotherapy has relative efficacy [11,12]; however, is typically not enough for severe cases [15] and mothers are hesitant to engage due to stigma [11]. Lastly, SSRIs often cause a range of side effects that impact compliance $[22,23]$. Women are often concerned about the effect on a breastfed child [24]. The low-risk profile and minimal side effects associated with TMS treatment offer a range of advantages for women and infant development.

The findings of seven studies for a total sample of 60 women were analysed. Only one paper involved a single randomized controlled trial, while most of the data synthesized came from uncontrolled trials and case studies, which inherently have an increased risk of bias. Thus, additional sham-controlled trials are needed to confirm the efficacy and safety of TMS for the treatment of PPD. Despite this methodological limitation, promising trends are demonstrated when analysing patient's response to TMS.

Out of the seven studies reviewed, six reported the majority of participants experienced a reduction in depressive symptoms $[4,50,56,57,59,60]$. Studies by Brock et al. [57] and Cox et al. [56] found a reduction in EPDS scores and reported $73.69 \%$ and $66.67 \%$ of participants achieved remission of depressive symptoms respectively. Garcia et al. [4] found a reduction in HRSD and CGI scores and reported $88.89 \%$ of participants achieved remission of depressive symptoms. The paper by Myczkowski et al. [50] was the only paper that incorporated sham TMS as a control measure. The active TMS group reported statistically significant differences in HDRS-17, GAS and CGI scores when comparing baseline to week 6 (two weeks after treatment). Results were not deemed statistically significant when comparing active versus sham TMS. However, the study was underpowered, and reduction percentages were notably larger for the active group.

The two case studies reported improvements in depressive symptoms for each of their participants [59,60]. Cohen et al. [59] found a reduction in YMRS and HDRS scores when comparing baseline to end of treatment. Similarly, Ogden [60] found a reduction in HDRS, BDI and VAS scores when assessing baseline to end of treatment. Ozmut et al. [58] was the only study that produced less than promising results. Only $26.67 \%$ of participants reported a 50\% decrease in HDRS-17 scores; $40 \%$ reported a 50\% decrease in HARS-14 scores, and $53.33 \%$ reported EDS-10 scores of less than 13 points. The inconsistent findings between papers may be due to differences in study design, the type of TMS administered, and patient characteristics.

The majority of the studies administered left DLPFC as their stimulation site $[4,50,56,57,60]$. One study administered right DLPFC and bilateral DLPFC TMS treatment [59], and one study did not specify the stimulation site [58]. All studies that reported the stimulation site were over the DLPFC. This coincides with research on neuroimaging of MDD, which presented hypoactivity of the left DLPFC and hyperactivity of the right 
DLPFC [39]. Thus confirming the ideal brain structure to be stimulated for the management of depressive symptoms.

Stimulus frequency varied widely between studies. Frequencies of 20, 10 and $5 \mathrm{~Hz}$ were used. Stimulus intensity varied from $120 \%$ to $80 \%$ of the resting motor threshold. The majority of the studies that reported high-frequency $(>5 \mathrm{~Hz})$ rTMS were over the left DLPFC, which concurs with homogeneous research that supports the specific use of high-frequency TMS to the left dorsolateral prefrontal cortex (DLPFC) for patients with treatment-resistant depression $[38,46]$. Nonetheless, the treatment protocol was inconsistent between studies and sometimes components of the protocol were not reported (i.e., number of trains, number of sessions), making it difficult to replicate these studies. Therefore, no conclusions could be formulated about optimal stimulus site, frequency or intensity for TMS treatment of PPD.

Papers analysed within this review were published within the last 15 years, with the exception of the case study by Odgen et al. [60]. Although the studies were published fairly recently, the overall quality of the study designs was poor. Each study had a small sample size $(n<20)$, and inconsistent inclusion criteria and study designs. Information about the participants' previous medical history, diagnosis, treatment history and treatment setting were often inadequately recorded. Many papers did not state what clinical measures were employed to determine their participant's diagnosis of PPD. It is difficult to interpret and generalise the data without certain pre-existing information. Moreover, outcome measures used to assess the efficacy of TMS were variable, with differences in the reduction of depressive symptoms possibly attributable to differences in the conceptualisation of depression across measures. Only two studies investigated social-relational outcomes and only another two studies investigated neuropsychological outcomes. Additional research is required to assess multiple outcomes, to achieve a holistic picture of the benefits of TMS treatment for PPD. Interestingly, the two case studies provide a stimulus for further research in TMS treatment for patients experiencing manic episodes and psychotic features respectively $[59,60]$.

The administration of TMS for the treatment of PPD is well tolerated. The studies, though having significant methodological flaws, provide early, encouraging evidence that TMS may be effective in PPD treatment, especially as many of the studies showed patients to have achieved remission rather than response, with no reported adverse side effects within the postpartum population. However, the lack of quality evidence (absence of randomised control trials, small sample sizes, and lack of homogeneity across treatment protocols) indicates the need for further research before any recommendation for TMS in treating PPD can be made.

There is growing research on the use of TMS in treating a range of psychiatric disorders, and this approach appears to revolutionise the way mental health disorders can be managed, due to the non-invasive technique and low side effect profile. A substantial body of evidence for the use of TMS in treating MDD has been established, however, research indicates PPD has a differential presentation and response to antidepressant treatment when compared to MDD outside the postpartum period. Hence, further research into the efficacy and acceptability of TMS for PPD is recommended.

Supplementary Materials: The following are available online at https:/ /www.mdpi.com/article / 10.3390 /psychiatryint2030020/s1.

Author Contributions: Conceptualization, M.M.H., S.C.C. and D.A.B.; methodology, M.M.H.; software, M.M.H.; validation, M.M.H., S.C.C. and D.A.B.; formal analysis, M.M.H.; investigation, M.M.H.; resources, M.M.H.; data curation, M.M.H.; writing-original draft preparation, M.M.H.; writingreview and editing, S.C.C. and D.A.B.; visualization, M.M.H.; supervision, S.C.C. and D.A.B.; project administration, S.C.C. and D.A.B. All authors have read and agreed to the published version of the manuscript.

Funding: This research received no external funding.

Institutional Review Board Statement: Not applicable. 
Informed Consent Statement: Not applicable.

Data Availability Statement: Not applicable.

Conflicts of Interest: The authors declare no conflict of interest.

\section{References}

1. Gavin, N.; Gaynes, B.; Lohr, K.; Meltzer-Brody, S.; Gartlehner, G.; Swinson, T. Perinatal Depression. Obstet. Gynecol. 2005, 106, 1071-1083. [CrossRef]

2. Almond, P. Postnatal depression: A global public health perspective. Perspect. Public Health 2009, 129, 221-227. [CrossRef] [PubMed]

3. Kirmayer, L.; Gómez-Carrillo, A. Agency, embodiment and enactment in psychosomatic theory and practice. Med. Humanit. 2019, 45, 169-182. [CrossRef] [PubMed]

4. Garcia, K.; Flynn, P.; Pierce, K.; Caudle, M. Repetitive transcranial magnetic stimulation treats postpartum depression. Brain Stimul. 2010, 3, 36-41. [CrossRef] [PubMed]

5. Ganho-Ávila, A.; Poleszczyk, A.; Mohamed, M.; Osório, A. Efficacy of Rtms in Decreasing Postnatal Depression Symptoms: A Systematic Review. Psychiatry Res. 2019, 279, 315-322. [CrossRef]

6. American Psychiatric Association. Diagnostic and Statistical Manual of Mental Disorders, 5th ed.; American Psychiatric Association: Washington, DC, USA, 2013.

7. Wisner, K.; Sit, D.; McShea, M.; Rizzo, D.; Zoretich, R.; Hughes, C.; Eng, H.; Luther, J.; Wisniewski, S.; Costantino, M.; et al. Onset Timing, Thoughts of Self-Harm, and Diagnoses in Postpartum Women with Screen-Positive Depression Findings. JAMA Psychiatry 2013, 70, 490. [CrossRef]

8. Woody, C.; Ferrari, A.; Siskind, D.; Whiteford, H.; Harris, M. A Systematic Review and Meta-Regression of The Prevalence and Incidence of Perinatal Depression. J. Affect. Disord. 2017, 219, 86-92. [CrossRef]

9. Semkovska, M.; McLoughlin, D. Objective Cognitive Performance Associated with Electroconvulsive Therapy for Depression: A Systematic Review and Meta-Analysis. Biol. Psychiatry 2010, 68, 568-577. [CrossRef]

10. Semkovska, M.; Noone, M.; Carton, M.; McLoughlin, D. Measuring Consistency of Autobiographical Memory Recall in Depression. Psychiatry Res. 2012, 197, 41-48. [CrossRef]

11. Guille, C.; Newman, R.; Fryml, L.; Lifton, C.; Epperson, C. Management of Postpartum Depression. J. Midwifery Womens Health 2013, 58, 643-653. [CrossRef] [PubMed]

12. Parsons, C.; Young, K.; Rochat, T.; Kringelbach, M.; Stein, A. Postnatal Depression and Its Effects on Child Development: A Review of Evidence from Low- and Middle-Income Countries. Br. Med. Bull. 2011, 101, 57-79. [CrossRef] [PubMed]

13. Sockol, L.; Epperson, C.; Barber, J. A Meta-Analysis of Treatments for Perinatal Depression. Clin. Psychol. Rev. 2011, 31, 839-849. [CrossRef] [PubMed]

14. van Ravesteyn, L.; Lambregtse-van den Berg, M.; Hoogendijk, W.; Kamperman, A. Interventions to Treat Mental Disorders During Pregnancy: A Systematic Review and Multiple Treatment Meta-Analysis. PLoS ONE 2017, 12, e0173397. [CrossRef] [PubMed]

15. MacQueen, G.; Frey, B.; Ismail, Z.; Jaworska, N.; Steiner, M.; Lieshout, R.; Kennedy, S.; Lam, R.; Milev, R.; Parikh, S.; et al. Canadian Network for Mood and Anxiety Treatments (CANMAT) 2016 Clinical Guidelines for the Management of Adults With Major Depressive Disorder. Can. J. Psychiatry 2016, 61, 588-603. [CrossRef]

16. De Crescenzo, F.; Perelli, F.; Armando, M.; Vicari, S. Selective Serotonin Reuptake Inhibitors (Ssris) for Post-Partum Depression (PPD): A Systematic Review of Randomized Clinical Trials. J. Affect. Disord. 2014, 152-154, 39-44. [CrossRef]

17. Sharma, V.; Sommerdyk, C.; Xie, B.; Campbell, K. Pharmacotherapy of bipolar II disorder during and after pregnancy. Curr. Drug Saf. 2013, 8, 246-252. [CrossRef]

18. Kim, D.; Epperson, C.; Weiss, A.; Wisner, K. Pharmacotherapy of Postpartum Depression: An Update. Expert Opin. Pharmacother. 2014, 15, 1223-1234. [CrossRef]

19. Thomson, M.; Sharma, V. Therapeutics of Postpartum Depression. Expert Rev. Neurother. 2016, 17, 495-507. [CrossRef]

20. Munk-Olsen, T.; Gasse, C.; Laursen, T. Prevalence of Antidepressant Use and Contacts with Psychiatrists and Psychologists in Pregnant and Postpartum Women. Acta Psychiatr. Scand. 2011, 125, 318-324. [CrossRef]

21. Pearlstein, T.; Zlotnick, C.; Battle, C.; Stuart, S.; O'Hara, M.; Price, A.; Grause, M.; Howard, M. Patient Choice of Treatment for Postpartum Depression: A Pilot Study. Arch. Womens Ment. Health 2006, 9, 303-308. [CrossRef]

22. Cassano, P.; Fava, M. Tolerability Issues During Long-Term Treatment with Antidepressants. Ann. Clin. Psychiatry 2004, 16, 15-25. [CrossRef] [PubMed]

23. Turner, K.; Sharp, D.; Folkes, L.; Chew-Graham, C. Women's views and experiences of antidepressants as a treatment for postnatal depression: A qualitative study. Fam. Pract. 2008, 25, 450-455. [CrossRef]

24. Kendall-Tackett, K.; Hale, T. Review: The Use of Antidepressants in Pregnant and Breastfeeding Women: A Review of Recent Studies. J. Hum. Lact. 2010, 26, 187-195. [CrossRef] [PubMed]

25. Kornstein, S.; Sloan, D.; Thase, M. Gender-specific differences in depression and treatment response. Psychopharmacol. Bull. 2002, 36, 99-112. [PubMed] 
26. Sloan, D.; Kornstein, S. Gender differences in depression and response to antidepressant treatment. Psychiatr. Clin. N. Am. 2003, 26, 581-594. [CrossRef]

27. Brummelte, S.; Galea, L. Depression during pregnancy and postpartum: Contribution of stress and ovarian hormones. Prog. Neuro-Psychopharmacol. Biol. Psychiatry 2010, 34, 766-776. [CrossRef]

28. Gutiérrez-Lobos, K.; Scherer, M.; Anderer, P.; Katschnig, H. The influence of age on the female/male ratio of treated incidence rates in depression. BMC Psychiatry 2002, 2, 3. [CrossRef]

29. Hammarström, A.; Lehti, A.; Danielsson, U.; Bengs, C.; Johansson, E. Gender-related explanatory models of depression: A critical evaluation of medical articles. Public Health 2009, 123, 689-693. [CrossRef]

30. Polanía, R.; Nitsche, M.; Ruff, C. Studying and Modifying Brain Function with Non-Invasive Brain Stimulation. Nat. Neurosci. 2018, 21, 174-187. [CrossRef]

31. Karsen, E.; Watts, B.; Holtzheimer, P. Review of the Effectiveness of Transcranial Magnetic Stimulation for Post-Traumatic Stress Disorder. Brain Stimul. 2014, 7, 151-157. [CrossRef] [PubMed]

32. Borgomaneri, S.; Battaglia, S.; Garofalo, S.; Tortora, F.; Avenanti, A.; di Pellegrino, G. State-Dependent TMS over Prefrontal Cortex Disrupts Fear-Memory Reconsolidation and Prevents the Return of Fear. Curr. Biol. 2020, 30, 3672-3679.e4. [CrossRef]

33. Borgomaneri, S.; Battaglia, S.; Avenanti, A.; Pellegrino, G. Don't Hurt Me No More: State-Dependent Transcranial Magnetic Stimulation for the Treatment of Specific Phobia. J. Affect. Disord. 2021, 286, 78-79. [CrossRef]

34. Herrmann, M.; Katzorke, A.; Busch, Y.; Gromer, D.; Polak, T.; Pauli, P.; Deckert, J. Medial Prefrontal Cortex Stimulation Accelerates Therapy Response of Exposure Therapy in Acrophobia. Brain Stimul. 2017, 10, 291-297. [CrossRef] [PubMed]

35. Trevizol, A.; Shiozawa, P.; Cook, I.; Sato, I.; Kaku, C.; Guimarães, F.; Sachdev, P.; Sarkhel, S.; Cordeiro, Q. Transcranial Magnetic Stimulation for Obsessive-Compulsive Disorder. J. ECT 2016, 32, 262-266. [CrossRef] [PubMed]

36. Carmi, L.; Alyagon, U.; Barnea-Ygael, N.; Zohar, J.; Dar, R.; Zangen, A. Clinical and Electrophysiological Outcomes of Deep TMS over the Medial Prefrontal and Anterior Cingulate Cortices in OCD Patients. Brain Stimul. 2018, 11, 158-165. [CrossRef] [PubMed]

37. Tran, B.; Ha, G.; Vu, G.; Nguyen, L.; Latkin, C.; Nathan, K.; McIntyre, R.; Ho, C.; Tam, W.; Ho, R. Indices of Change, Expectations, and Popularity of Biological Treatments for Major Depressive Disorder between 1988 and 2017: A Scientometric Analysis. Int. J. Environ. Res. Public Health 2019, 16, 2255. [CrossRef] [PubMed]

38. Carpenter, L.; Janicak, P.; Aaronson, S.; Boyadjis, T.; Brock, D.; Cook, I.; Dunner, D.L.; Lanocha, K.; Solvason, H.B.; Demitrack, M.A. Transcranial magnetic stimulation (TMS) for major depression: A multisite, naturalistic, observational study of acute treatment outcomes in clinical practice. Depress. Anxiety 2012, 29, 587-596. [CrossRef] [PubMed]

39. Taïb, S.; Arbus, C.; Sauvaget, A.; Sporer, M.; Schmitt, L.; Yrondi, A. How Does Repetitive Transcranial Magnetic Stimulation Influence the Brain in Depressive Disorders? J. ECT 2018, 34, 79-86. [CrossRef] [PubMed]

40. Benadhira, R.; Thomas, F.; Bouaziz, N.; Braha, S.; Andrianisaina, P.; Isaac, C.; Moulier, V.; Januel, D. A Randomized, ShamControlled Study of Maintenance Rtms for Treatment-Resistant Depression (TRD). Psychiatry Res. 2017, 258, 226-233. [CrossRef]

41. Bulteau, S.; Sébille, V.; Fayet, G.; Thomas-Ollivier, V.; Deschamps, T.; Bonnin-Rivalland, A.; Laforgue, E.; Pichot, A.; Valrivière, P.; Auffray-Calvier, E.; et al. Efficacy of Intermittent Theta Burst Stimulation (Itbs) and 10-Hz High-Frequency Repetitive Transcranial Magnetic Stimulation (Rtms) in Treatment-Resistant Unipolar Depression: Study Protocol for a Randomised Controlled Trial. Trials 2017, 18, 1-10. [CrossRef]

42. Lefaucheur, J.; Aleman, A.; Baeken, C.; Benninger, D.; Brunelin, J.; Di Lazzaro, V.; Filipović, S.; Grefkes, C.; Hasan, A.; Hummel, F.; et al. Evidence-Based Guidelines on the Therapeutic Use of Repetitive Transcranial Magnetic Stimulation (Rtms): An Update (2014-2018). Clin. Neurophysiol. 2020, 131, 474-528. [CrossRef] [PubMed]

43. Miron, J.; Voetterl, H.; Mansouri, F.; Blumberger, D.; Daskalakis, Z.; Downar, J. A Case Series of a Novel 1 Hz Right-Sided Dorsolateral Prefrontal Cortex Rtms Protocol in Major Depression. Brain Stimul. 2020, 13, 372-374. [CrossRef]

44. Sehatzadeh, S.; Daskalakis, Z.; Yap, B.; Tu, H.; Palimaka, S.; Bowen, J.; O’Reilly, D. Unilateral and Bilateral Repetitive Transcranial Magnetic Stimulation for Treatment-Resistant Depression: A Meta-Analysis of Randomized Controlled Trials over 2 Decades. J. Psychiatry Neurosci. 2019, 44, 151-163. [CrossRef]

45. Berlim, M.; Van den Eynde, F.; Daskalakis, Z. A Systematic Review and Meta-Analysis on the Efficacy and Acceptability of Bilateral Repetitive Transcranial Magnetic Stimulation (Rtms) for Treating Major Depression. Psychol. Med. 2012, 43, 2245-2254. [CrossRef]

46. Perera, T.; George, M.; Grammer, G.; Janicak, P.; Pascual-Leone, A.; Wirecki, T. The Clinical TMS Society Consensus Review and Treatment Recommendations for TMS Therapy for Major Depressive Disorder. Brain Stimul. 2016, 9, 336-346. [CrossRef]

47. Aarre, T.; Dahl, A.; Johansen, J.; Kjønniksen, I.; Neckelmann, D. Efficacy of repetitive transcranial magnetic stimulation in depression: A review of the evidence. Nord. J. Psychiatry 2003, 57, 227-232. [CrossRef] [PubMed]

48. Slotema, C.; Blom, J.; Hoek, H.; Sommer, I. Should We Expand the Toolbox of Psychiatric Treatment Methods to Include Repetitive Transcranial Magnetic Stimulation (rTMS)? J. Clin. Psychiatry 2010, 71, 873-884. [CrossRef]

49. Peng, L.; Fu, C.; Xiong, F.; Zhang, Q.; Liang, Z.; Chen, L.; He, C.; Wei, Q. Effects of repetitive transcranial magnetic stimulation on depression symptoms and cognitive function in treating patients with postpartum depression: A systematic review and meta-analysis of randomized controlled trials. Psychiatry Res. 2020, 290, 113124. [CrossRef] 
50. Myczkowski, M.; Dias, A.; Luvisotto, T.; Arnaut, D.; Bellini, B.; Marcolin, M.; Mansur, C.G.; Rennó, J.; de Souza, G.T.; Ribeiro, P.L. Effects of repetitive transcranial magnetic stimulation on clinical, social, and cognitive performance in postpartum depression. Neuropsychiatr. Dis. Treat. 2012, 8, 491-500. [CrossRef]

51. Moher, D.; Liberati, A.; Tetzlaff, J.; Altman, D. Preferred reporting items for systematic reviews and meta-analyses: The PRISMA statement. J. Clin. Epidemiol. 2009, 62, 1006-1012. [CrossRef] [PubMed]

52. Crowe, M.; Sheppard, L. A general critical appraisal tool: An evaluation of construct validity. Int. J. Nurs. 2011, 48, 1505-1516. [CrossRef]

53. Crowe, M. Crowe Critical Appraisal Tool (CCAT) User Guide; Conchra House: Scotland, UK, 2013.

54. Crowe, M.; Sheppard, L. A review of critical appraisal tools show they lack rigor: Alternative tool structure is proposed. J. Clin. Epidemiol. 2011, 64, 79-89. [CrossRef]

55. Crowe, M.; Sheppard, L.; Campbell, A. Comparison of the effects of using the Crowe Critical Appraisal Tool versus informal appraisal in assessing health research: A randomised trial. Int. J. Evid. Based Healthc. 2011, 9, 444-449. [CrossRef]

56. Cox, E.; Killenberg, S.; Frische, R.; McClure, R.; Hill, M.; Jenson, J.; Pearson, B.; Meltzer-Brody, S. Repetitive transcranial magnetic stimulation for the treatment of postpartum depression. J. Affect. Disord. 2020, 264, 193-200. [CrossRef]

57. Brock, D.; Demitrack, M.; Groom, P.; Holbert, R.; Rado, J.; Gross, P.; Goethe, J.W.; Schrodt, G.R.; Weeks, H.R. Effectiveness of NeuroStar transcranial magnetic stimulation (TMS) in patients with major depressive disorder with postpartum onset. Brain Stimul. 2016, 9, e7. [CrossRef]

58. Ozmut, O.; Balibey, H.; Yilan, Y.; Algul, A.; Ebrinc, S.; Cetin, M.; Tutuncu, R.; Ates, A. Repetitive transcranial magnetic stimulation for the treatment of depression during pregnancy and postpartum period. Bull. Clin. Psychopharmacol. 2015, 25, S203.

59. Cohen, R.; Ferreira, M.; Ferreira, M.; Fregni, F. Use of repetitive transcranial magnetic stimulation for the management of bipolar disorder during the postpartum period. Brain Stimul. 2008, 1, 224-226. [CrossRef]

60. Ogden, M.; Lyndon, W.; Pridmore, S. Repetitive transcranial magnetic stimulation (rTMS) in major depressive episode with postpartum onset-A case study. German J. Psychiatry 1999. Available online: http:/ / ecite.utas.edu.au/17760 (accessed on 1 May 2021).

61. Lisanby, S.; Gutman, D.; Luber, B.; Schroeder, C.; Sackeim, H. Sham TMS: Intracerebral measurement of the induced electrical field and the induction of motor-evoked potentials. Biol. Psychiatry 2001, 49, 460-463. [CrossRef]

62. Beck, A. An Inventory for Measuring Depression. Arch. Gen. Psychiatry 1961, 4, 561-571. [CrossRef]

63. Cox, J.; Holden, J.; Sagovsky, R. Detection of Postnatal Depression. Br. J. Psychiatry 1987, 150, 782-786. [CrossRef]

64. Spielberger, C. Manual for the State-Trait Anxiety Inventory: STAI (Form Y); Consult. Psychol Press: Palo Alto, CA, USA, 1983.

65. Hamilton, M. Development of a Rating Scale for Primary Depressive Illness. Br. J. Soc. Clin. Psychol. 1967, 6, 278-296. [CrossRef]

66. Guy, W. ECDEU Assessment Manual for Psychopharmacology; National Institute of Mental Health, US Department of Health, Education, and Welfare: Rockville, MD, USA, 1976.

67. Blacker, D. Psychiatric rating scales. In Compr Textbook Psychiatry, 7th ed.; Sadock, B., Sadock, V., Eds.; Lippincott Williams \& Wilkins: Philadelphia, PA, USA, 2000.

68. Mansur, C.; Myczkowki, M.; de Barros Cabral, S.; Sartorelli, M.; Bellini, B.; Dias, Á.; Bernik, M.A.; Marcolin, M.A. Placebo effect after prefrontal magnetic stimulation in the treatment of resistant obsessive-compulsive disorder: A randomized controlled trial. Int. J. Neuropsychopharmacol. 2011, 14, 1389-1397. [CrossRef]

69. Hamilton, M. The Assessment of Anxiety States by Rating. Br. J. Med. Psychol. 1959, 32, 50-55. [CrossRef]

70. Campolina, A.; Ciconelli, R. SF-36 and the development of new quality of life assessment measures [SF-36 and the development of new assessment tools for quality of life]. Acta Reum. Port. 2008, 33, 127-133.

71. Cline, M.; Herman, J.; Shaw, E.; Morton, R. Standardization of the Visual Analogue Scale. Nurs. Res. 1992, 41, 378-379. [CrossRef]

72. Young, R.; Biggs, J.; Ziegler, V.; Meyer, D. A Rating Scale for Mania: Reliability, Validity and Sensitivity. Br. J. Psychiatry 1978, 133, 429-435. [CrossRef]

73. Brockington, I.; Oates, J.; George, S.; Turner, D.; Vostanis, P.; Sullivan, M.; Loh, C.; Murdoch, C. A Screening Questionnaire for mother-infant bonding disorders. Arch. Womens Ment. Health 2001, 3, 133-140. [CrossRef]

74. Brockington, I.; Fraser, C.; Wilson, D. The Postpartum Bonding Questionnaire: A validation. Arch. Womens Ment. Health 2006, 9 , 233-242. [CrossRef]

75. Gorenstein, C.; Moreno, R.; Bernik, M.; Carvalho, S.; Nicastri, S.; Cordás, T.; Camargo, A.P.P.; Artes, R.; Andrade, L. Validation of the Portuguese version of the Social Adjustment Scale on Brazilian samples. J. Affect. Disord. 2002, 69, 167-175. [CrossRef]

76. Folstein, M.; McHugh, S. 'Mini-Mental State' a practical method for grading the cognitive state of patients for the clinician. $J$. Psychiatr. Res. 1975, 12, 189-198. [CrossRef]

77. Lezak, M. Neuropsychological Assessment, 3rd ed.; Oxford University Press: New York, NY, USA, 1995.

78. Rao, S. Cognitive Function Study Group of the National Multiple Sclerosis Society. A Manual for the Brief Repeatable Battery of Neuropsychological Tests in Multiple Sclerosis; National Multiple Sclerosis Society: New York, NY, USA, 1990.

79. Spreen, S.; Strauss, E. A Compendium of Neuropsychological Tests: Administration, Norms, and Commentary, 3rd ed.; Oxford University Press: Oxford, UK, 1991. 